HNO 2014 · 62:322-323

DOI 10.1007/s00106-014-2853-1

Online publiziert: 16. März 2014

๑) Springer-Verlag Berlin Heidelberg 2014

\title{
T. Deitmer
}

HNO-Klinik, Klinikum Dortmund

\section{HNO für jedes Lebensalter}

\section{Jahresversammlung der Deutschen Gesellschaft für Hals-, Nasen-, Ohren-Heilkunde, Kopf- und Halschirurgie in Dortmund, 28. Mai - 1. Juni 2014}

Sehr geehrte Leserinnen und Leser,

freundlicherweise räumt die Schriftleitung dieses wissenschaftlichen Publikationsorgans der Deutschen Gesellschaft für HNO-Heilkunde, Kopf- und Halschirurgie dem jeweiligen Präsidenten des Jahres ein, Beiträge zu Schwerpunktthemen der anstehenden Jahresversammlung in einem Heft im Sinne einer Mitherausgeberschaft zu publizieren.

Das Thema der Jahresversammlung in Dortmund lautet „HNO für jedes Lebensalter", wobei typischerweise einerseits das Kindesalter und andererseits das Seniorenalter von besonderem Interesse sind. Für das Kindesalter werden Kongressreferate gehalten und anderenorts publiziert. Interessante Beiträge für das Seniorenalter werden in Form von Hauptvorträgen gebracht, und ich freue mich, dass wir die Möglichkeit haben, die Expertise einiger dieser Redner durch eine Publikation hier festhalten und nachlesbar machen zu können.

Die Problematik der Dysphagie ist v. a. im fortgeschrittenen Lebensalter gegeben, sei es durch neurologische Erkrankungen oder durch Folgen und Nebenwirkungen multimodaler onkologischer Therapie von Kopf-Hals-Tumoren. Die zunehmende Nachfrage nach Diagnostik und Behandlung gilt es kompetent zu befriedigen, wobei ich mit Sorge wahrnehme, dass einerseits Neurologen und Geriater sich in dieses fachfremde Feld wagen, die endoskopische Diagnostik teilweise sogar auf nichtärztliches Personal delegiert wird, aber andererseits auch gerade im Bereich der HNO-Ärzte die Methode der Schluckendoskopie („functional endoscopic evaluation of swallowing", FEES) nur zögerlich angenommen und ins Routinerepertoire genommen wird. Ich freue mich, dass Frau Dr. Schröter-Morasch und Frau Dr. Graf als Phoniaterinnen und hervorragende Expertinnen in diesem Feld uns zur systematischen Vermittlung dieser Methode eine kompakte und praxisnahe Publikation zur Verfügung gestellt haben.

》) Die Problematik

der Dysphagie ist v. a.

im fortgeschrittenen

Lebensalter gegeben

Ein weiteres, auch im politischen Raum vielfach diskutiertes Thema ist die Palliativmedizin, die auch typischerweise für ältere Patienten infrage kommt. In der technisierten Medizin, in der ,alles geheilt werden kann", geht der Blick darauf verloren, dass wir als Ärzte nicht nur kurieren, sondern auch lindern müssen. Dieser Gedankenansatz der Palliation erfordert nicht allein eine intensive menschliche Zuwendung, sondern auch ,hartes“ Wissen und Erfahrung. Herr PD Dr. Büntzel öffnet seinen reichen Wissens- und Erfahrungsschatz zu diesem Thema.

Allein während meiner beruflichen Tätigkeitszeit hat sich die Haltung zur Onkochirurgie im Alter massiv gewandelt. 
Gestützt durch entsprechende Möglichkeiten der Anästhesiologie können immer mehr Patienten auch in höherem Lebensalter erfolgreich operiert werden. Herr Prof. Teymoortash legt eine aussagekräftige Studie zu chirurgischen und onkologischen Ergebnissen vor, die die Tendenz stützt, auch älteren Patienten ein Therapiekonzept mit Chirurgie anzubieten.

Ein alltägliches Erlebnis ist es, dass $\mathrm{Pa}$ tienten im Alter aus verschiedensten Indikationen Medikamente nehmen müssen, die die humorale Blutgerinnung oder die Thrombozytenaggregation hemmen. Die hergebrachte Haltung, für jede Art von Operation wegen der Blutungsgefahr eine Unterbrechung dieser Medikationen zu fordern, wird dem medizinischen Gesamtaspekt des Patienten nicht gerecht und ist überholt. Durch Absetzen solcher Medikamente kann sich das Risiko einer lebensbedrohenden kardiovaskulären Komplikation als wesentlich höher darstellen als das einer operativen Nachblutung. Für andere Fachgebiete existieren Studien und Reviews, die diese bilaterale Risikoabschätzung beleuchten. Für die HNO-Heilkunde, Kopf- und Halschirurgie existiert wenig publizierte Erkenntnis. Herr PD Dr. Knopf hat sich dieser teils interdisziplinären Aufgabe gestellt und zusätzlich eine umfassende Originalarbeit aus der HNO-Klinik des Klinikums rechts der Isar vorgelegt.

Ich wünsche Ihnen eine interessante und erkenntnisreiche Lektüre mit praxisnahen Aspekten für Ihre tägliche verantwortungsvolle Arbeit.

Ihr

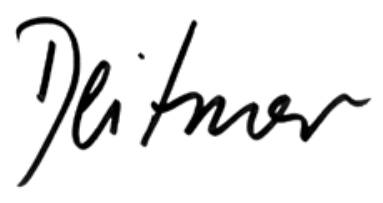

T. Deitmer

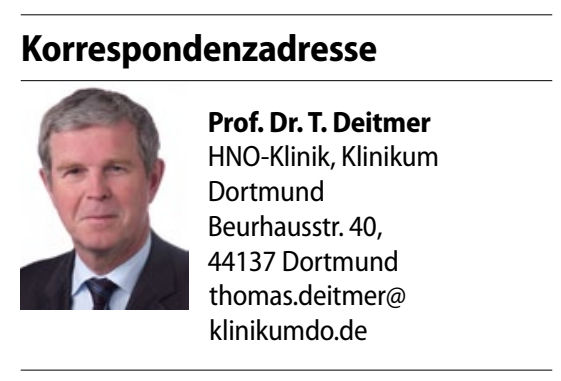

Interessenkonflikt. T. Deitmer gibt an, dass kein Interessenkonflikt besteht. 